IOPScience

\title{
VLBI Non-detection of a Candidate Dual AGN in a Galaxy Merger
}

Sándor Frey1 (iD), Davide Lena 2,3 (D), Peter G. Jonker2,3,

Krisztina É. Gabányi ${ }^{1,4}$ iD, and Zsolt Paragi ${ }^{5}$

Published 2019 January 2 • (c) 2019. The American Astronomical Society. All rights reserved.

Research Notes of the AAS, Volume 3, Number 1

frey.sandor@csfk.mta.hu

1 Konkoly Observatory, MTA CSFK, Konkoly út 15-17, H-1121 Budapest, Hungary

2 SRON, Netherlands Institute for Space Research, Sorbonnelaan 2, NL-3584 CA Utrecht, The Netherlands

3 Department of Astrophysics/IMAPP, Radboud University, P.O. Box 9010, NL-6500 GL Nijmegen, The Netherlands

${ }^{4}$ MTA-ELTE Extragalactic Astrophysics Research Group, Pázmány sétány 1/A, H-1117 Budapest, Hungary

5 Joint Institute for VLBI ERIC, Oude Hoogeveensedijk 4, NL-7991 PD

Dwingeloo, The Netherlands

Sándor Frey (D) https://orcid.org/0000-0003-3079-1889

Davide Lena (iD https://orcid.org/0000-0003-4184-6152

Krisztina É. Gabányi iD https://orcid.org/0000-0003-1020-1597

Zsolt Paragi (iD https://orcid.org/0000-0002-5195-335X 
Received 2018 December 21

Accepted 2018 December 28

Published 2019 January 2

Sándor Frey et al 2019 Res. Notes AAS 31

https://doi.org/10.3847/2515-5172/aafbOf

galaxies: individual (SDSS J085312.85+162616.0, DSS

J085312.34+162619.4); radio continuum: galaxies

Export citation and abstract

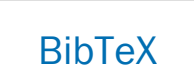

RIS

Numerical hydrodynamical simulations (e.g., Capelo et al. 2015) show that nuclear activity is experienced in multiple episodes during major galaxy mergers. Simultaneous activity in both nuclei becomes most likely at separations $\lesssim 10 \mathrm{kpc}$. These systems where the central supermassive black holes (SMBHs) are still gravitationally unbound are called dual active galactic nuclei (AGNs). Characterizing a large sample of dual AGNs would help constraining the merger rate of galaxies and SMBHs, understanding the role of galaxy mergers in triggering AGN activity, and making predictions for $\mathrm{SMBH}$ coalescence producing gravitational waves. However, securely identified dual AGNs are rare. Confirmation of each case would ideally require AGN indicators at multiple wavebands. Although radio emission is associated with a minority of AGNs, the very long baseline interferometry (VLBI) technique plays a unique role in confirming emission from dual AGNs, owing to its high angular resolution (e.g., An et al. 2018).

Based on optical and X-ray imaging and spectroscopy, Lena et al. (2018) discovered a dual AGN candidate hosted in interacting galaxies. At their redshift $z=0.064$, the 8 " 1 angular separation between the two objects (S1: SDSS J085312.85+162616.0 and S2: SDSS J085312.34+162619.4) 
corresponds to $11 \mathrm{kpc}$ projected linear distance. The analysis of multiband data collected so far indicates that S1 certainly harbours an AGN. The nature of S2 is less clear, with indications that the X-ray emission comes from starburst activity.

In the radio, the catalog of the $1.4 \mathrm{GHz}$ FIRST (White et al. 1997) survey contains a resolved source $\left(\right.$ R.A. $=08^{\mathrm{h}} 53^{\mathrm{m}} 12$ s 467 , decl. $\left.=+16^{\circ} 26^{\prime} 19^{\prime \prime} 19\right)$ with a peak brightness $2.75 \mathrm{mJy}^{\text {beam }}{ }^{-1}$ and integrated flux density $S_{1.4}=5.81 \mathrm{mJy}$. The radio source lies between the two optical/X-ray objects, closer to S2 (within $2^{\prime \prime)}$. Its deconvolved size is $8 " 1 \times 3$ " 4 , with a major axis position angle $116^{\circ}$ that roughly coincides with the S1-S2 position angle, suggesting that both may contribute to the radio emission.

We observed this radio source at three orders of magnitude higher angular resolution with the European VLBI Network (EVN) to check if one or both galaxies host a weak radio-emitting AGN. Both S1 and S2 fell in the undistorted field of view of our observation. The $1.7 \mathrm{GHz}$ EVN experiment took place in e-VLBI mode on 2018 November 21 and lasted for $2 \mathrm{hr}$. The radio telescopes were Cambridge, Darnhall, Defford, Jodrell Bank Mk2 (UK), Medicina, Sardinia (Italy), Effelsberg (Germany), Hartebeesthoek (South Africa), Onsala (Sweden), and Tianma (China). The correlator integration time was $1 \mathrm{~s}$. Eight times 32 frequency channels were used with $128 \mathrm{MHz}$ total bandwidth in both left and right circular polarizations. We applied phase-referencing to a nearby (1.52) compact calibrator J0856+1739, and also observed OJ 287 as fringe-finder. The total time spent on the target was $1.6 \mathrm{hr}$. We used the Astronomical Image Processing System (Greisen 2003) to calibrate the EVN data. Imaging was attempted in the Difmap program (Shepherd et al. 1994). A more detailed description of the procedure can be found in e.g., Frey et al. (2013). 
We used uniform weighting to minimize the image noise. With a resolution of $\sim 4$ mas, no compact radio features were detected, neither around the position of the FIRST source, nor at S1 and S2. The $6 \sigma$ upper limit for the brightness is $0.13 \mathrm{mJy} \mathrm{beam}^{-1}$. The radio emission detected in FIRST $\left(S_{1.4}=5.81 \mathrm{mJy}\right.$, power $\left.P_{1.4}=5.4 \times 10^{22} \mathrm{~W} \mathrm{~Hz}^{-1}\right)$ is apparently resolved out with VLBI and may originate from spatially extended starburst activity in the merging galaxies or resolved AGN-related structures. Assuming no AGN, the corresponding star formation rate is estimated as $\sim 30 M_{\odot} \mathrm{yr}^{-1}$ (Hopkins et al. 2003) in the system, about three times higher than calculated by Lena et al. (2018) from $u$-band optical magnitudes.

Based on our short EVN observations, we are unable to fully exclude the presence of one or two low-luminosity AGNs in the S1/S2 system. A flux density $S \approx 0.1 \mathrm{mJy}$ in a hypothetical compact source that would have remained undetected in our EVN experiment corresponds to $P_{1.4} \approx 10^{21} \mathrm{~W} \mathrm{~Hz}^{-1}$ at $z=0.064$. This exceeds the average power $2 \times 10^{20}$ $\mathrm{W} \mathrm{Hz}{ }^{-1}$ for individual radio supernovae (Bondi et al. 2005), but is below the radio power of the weakest AGNs studied in the COSMOS field (Smolčić et al. 2008).

The EVN is a joint facility of independent European, African, Asian, and North American radio astronomy institutes. Scientific results from data presented in this publication are derived from the project RSF09. The research leading to these results has received funding from the European Commission Horizon 2020 Research and Innovation Programme under grant agreement No. 730562 (RadioNet). This work was supported by the NKFIH-OTKA NN110333 grant. K.É.G. acknowledges the Bolyai Research Scholarship of the Hungarian Academy of Sciences. 


\section{References}

卫 An T., Mohan P. and Frey S. 2018 RaSc 531211 Crossref ADS

^ Bondi M., Pérez-Torres M.-A., Dallacasa D. and Muxlow T. W. B. 2005 MNRAS 361748

Crossref ADS

^ Capelo P. R., Volonteri M., Dotti M. et al 2015 MNRAS 4472123 Crossref ADS

卫 Frey S., Paragi Z., Gabányi K. É. and An T. 2013 A\&A 552109 Crossref ADS

卫 Greisen E. W. 2003 Information Handling in Astronomy: Historical Vistas, Vol. 285 ed A. Heck (Dordrecht: Kluwer) 109

Crossref ADS

^ Hopkins A. M., Miller C. J., Nichol R. C. et al 2003 ApJ 599971 IOPscience (http://iopscience.iop.org/0004-637X/599/2 /971) ADS

卫 Lena D., Panizo-Espinar G., Jonker P. G., Torres M. A. P. and Heida M. 2018 MNRAS 4781326

Crossref ADS

卫 Shepherd M. C., Pearson T. J. and Taylor G. B. 1994 BAAS 26987 ADS

1 Smolčić V., Schinnerer E., Scodeggio M. et al2008 ApJS 17714 IOPscience (http://iopscience.iop.org/0067-0049/177/1 114) ADS

^ White R. L., Becker R. H., Helfand D. J. and Gregg M. D. 1997 ApJ 475 
479

IOPscience (http://iopscience.iop.org/0004-637X/475/2

/479) ADS

\begin{tabular}{lll} 
Export references: & BibTeX RIS \\
\hline
\end{tabular} 